\title{
Endovascular management of complicated IgG4 periaortitis
}

\author{
Mohamed Elsherif ${ }^{1}$, Wael Tawfick ${ }^{1}$, Niamh Hynes ${ }^{2}$ and Sherif Sultan ${ }^{1,2 *}$ \\ ${ }^{1}$ Western Vascular Institute, Department of Vascular and Endovascular Surgery, Galway University Hospital, National University of Ireland, Galway, Ireland \\ ${ }^{2}$ Department of Vascular and Endovascular Surgery, Galway Clinic, Ireland
}

\begin{abstract}
We report on an endovascular management of complicated IgG4 peri-aortitis after failed medical treatment. A uni-modular Aorto-Bi-iliac device was used. At Follow up, the aortic sac showed complete Remodelling with total resolution of the chronic abdominal pain. This case illustrates an alternative modality in management of complicated periaortic inflammation.
\end{abstract}

\section{Case report}

A 56 years old gentleman with a background history of hypertension, type II diabetes mellitus on oral hypoglycaemic tablets. He was managed with bilateral double J ureteric stent. He presented to our service complaining of non specific abdominal and back pain. Computerised Tomography (CT) scan demonstrated an incidental finding of retroperitoneal fibrosis around the aorta, both iliac arteries and ureters (Figure 1a). He had elevated Erythrocyte sedimentation rate and $\mathrm{C}$ - reactive protein. He was commenced on oral steroids for retroperitoneal fibrosis. Initially, he responded to steroid therapy. At Six months, pain was constant and affecting patient's quality of life. His IgG 4 was elevated. CT guided retroperitoneal biopsy to histologically confirm the cause of this retroperitoneal fibrosis were performed (Figure 1b). Histopathology revealed spindle cells with mature collagen cells with scattered lymphocytes, confrimung the diagnosis of retroperitoneal fibrosis, with no evidence of malignanacy.

The patient started to deteriorate with worsening abdominal and back pain unresponsive to opiates. Clinically, the patient was feeling unwell, with pyrexia and uncontrolled hyperglycaemia requiring both insulin and oral hypoglycaemic drugs. Bloods showed an increase in the inflammatory markers and erythrocyte sedimentation rate. Septic screen to identify a source of infection was negative. As he was not responding to mono-steroid therapy, azathioprine and Doxacycline were commemced.
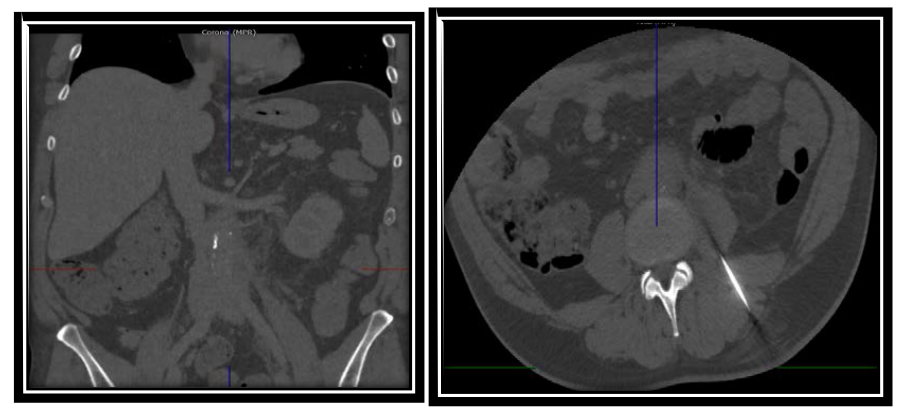

A

Figure 1. A. CT abdomen demonstrate increase periaortic inflammation with irregular borders with intramural hematoma. B. CT guided retroperitoneal biopsy showed the needle passing through the paraspinal muscle for retroperitoneal biopsy.
The patient continued to deteriorate and was hospitalised for investigation for fever of unknow etiology. Repeat CT scan angiogram revealed an expanding periaortic inflammation measuring $40 \mathrm{~mm}$ in diamter across the whole lenghth of the infra renal aorta, with an aortic haematoma extending from the left accessory renal artery down to the aortic bifurcation.

A decision was taken to proceed with endovascular mangement, due to failure of medical therapy and the presence of the extensive peri-aortic fibrosis and inflammation. An endovascular aorto bi-iliac stent graft AFX (Endologix, Irvine, CA, U.S) was deployed. Both iliac limbs were anchored by two Palmaz Genesis balloon expandable stents (Cordis, Fermont, CA, U.S).

The patient experienced an uneventful postoperative course. He was commenced on aspirin. His inflammatory markers dramatically improved. At six weeks follow-up, patient's symptoms had completely disappeared. His blood glucose level was controlled only on oral hypoglycaemic drugs, without the need for insulin and he was off of all of his pain medications.

At 6 months follow up CT angiography revealed a patent stent, complete aortic remodelling, with total resolving of the peri-aortic fibrosis (Figure 2). At 12 months, duplex follow up scan and vascular laboratory physiological parameters were entirly normal.

\section{Discussion}

Chronic periaortitis is a rare disease, occuring in middle-aged adult males and is characterised by fibrotic reaction surrounding the abdominal aorta with adventitial and periadventitial inflammation, medial thinning, and a chronic retroperitoneal inflammatory process

Correspondence to: Prof. Sherif Sultan, Professor of Vascular and Endovascular Surgery, Western Vascular Institute, Clincal Vascular Lead Soalta Hospital Group, Department of Vascular and Endovascular Surgery, University Hospital Galway, National University of Ireland, Galway, Ireland, Tel: +35391 720121; Fax: +35391720122; E-mail: sherif.sultan@hse.ie

Key words: aortic remodelling, endovascular, inflamatory AAA, IgG4 periaortitis Received: January 19, 2016; Accepted: February 09, 2016; Published: February 11,2016 


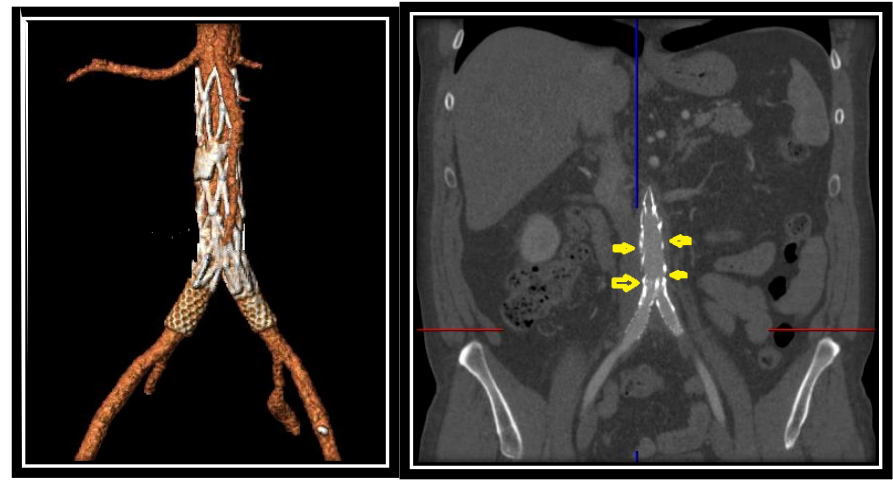

Figure 2. Post EVAR CT demonstrate patent stents, complete aortic remodelling and resolving peri-aortic inflammation.

[1]. The endovascular management of complicated peri-aortic inflammation is a challenging task and there are not any fixed guideline or algorithm to follow after medical mangement failure.

Immunoglobulin G-4 (IgG4) - related aortitis is the most common type of retroperitoneal fibrosis, mostly affecting the infrarenal part of the abdominal aorta and iliacs arteries, with inflitrates around the inferior vena cava and ureters [2].

IgG4-related aortic lesions include aortits, periaortitis, inflammatory abdominal aortic aneurysm (AAA), and retroperitoneal fibrosis, it is sometimes difficult to clearly distinguish these entities [1]. Corticosteroids may modulate the thickened aortic walls and the dense periaortic fibrous tissues. However, the effectiveness of corticosteroid therapy for IgG4-related aortic lesions remains controversial [3]. In cases where aortitis are refractory to the combination of steroids and immunosuppressive therapy, there is a need for a biopsy, to clarify the diagnosis and rule out other sinister aetiologies.

Our patient was primary managed conservatively with corticosteroids. Initially, the symptoms and the signs were controlled, however the abdominal pain was excoriating which demanded a CT guided retroperitoneal biopsy. The patient's symptoms and signs flared up and became refractory to all modalities of medical therapy. A dramatic increase in periaortic tissue with an intramural hematoma was observed. We postulated that this could either be due to an inflammatory response secondary to the $\mathrm{CT}$ guided retroperitoneal biopsy or due to mechanical disruption of the aortic wall by the needle.

An open surgical approach for mangement of an IgG4-related inflammatory AAA is associated with high morbidity due to the extensive adhesions of the periaortic tissues. An endovascular approach helps avoid extensive dissection, thus minimising the risk of morbidity and mortality.

Ikeda, et al, reported on a case of endovascular repair of a false aneurysm arising at the suprarenal part of the aorta as a consequence of IgG4-related periaortitis. This was associated with pancreatitis and cholangitis. One year follow-up, revealed complete resolution of periaortic surrounding inflammation [4].

Endovascular repair is more suitable for inflammatory AAAs as the luminal diameter of periaortitis is near normal and a false aneurysm is caused by disruption of part of the aortic wall [5].

This case demonstrates the role of endovascular stenting in preventing aortic wall expansion and promote aortic remodelling. CT showed complete suppression of aortic inflammation and complete aortic remodelling within three months.

Our case supplements prevoius scanty reports of endovascular management of complicated infrarenal peri-aortic retroperitoneal fibrosis due to IgG4-related periaortitis.

Retroperitoneal fibrosis carries a high risk of developing chronic kidney disease due to ureteric compression and obstruction for which it will require uretric stents or uretrolysis [6]. Lower limb claudication may occur as a result of common iliac artery compression or stenosis [7]. Hapka et al. reported three years follow up of a case of retroperitoneal fibrosis associated with right hydronephrosis, right common iliac artery stenosis and saccular aneurysm, which was managed successfully with endovascular graft [7].

\section{Conclusion}

Corticosteroid therapy is effective for uncomplicated IgG4-related periaortitis. In cases where medical therapy fails, endovascular surgery provides a promising outcome.

\section{References}

1. Stone JR (2011) Aortitis, periaortitis, and retroperitoneal fibrosis, as manifestations of IgG4-related systemic disease. Curr Opin Rheumatol 23: 88-94.[Crossref]

2. vanBommel EF, Jansen I, Hendriksz TR, Aarnoudse AL (2009) Idiopathic retroperitoneal fibrosis: prospective evaluation of incidence and clinicoradiologic presentation. Medicine (Baltimore) 88: 193-201.[Crossref]

3. Kasashima S, Zen Y, Kawashima A, Endo M, Matsumoto Y, et al. (2009) A new clinicopathological entity of IgG4-related inflammatory abdominal aortic aneurysm. $J$ Vasc Surg 49: 1264-1271.[Crossref]

4. Ikeda A, Mitomi K, Konishi T, Matsuzaki K, Jikuya T, et al. (2015) Endovascular Repair of a False Aneurysm Developing from IgG4-Related Periaortitis during Corticosteroid Therapy. Ann Vasc Surg 29: 1452. [Crossref]

5. Paravastu SC, Ghosh J, Murray D, Farquharson FG, Serracino-Inglott F, et al. (2009) A systematic review of open versus endovascular repair of inflammatory abdominal aortic aneurysms. Eur J Vasc Endovasc Surg 38: 291-297.[Crossref]

6. Ha YJ, Jung SJ, Lee KH, Lee SW, Lee SK, et al. (2011) Retroperitoneal fibrosis in 27 Korean patients: single center experience. J Korean Med Sci 26: 985-990.[Crossref]

7. Hapka L, Halena G, Brzezi Å, Ski M (2014) Retroperitoneal fibrosis as a rare cause of lower limb claudication. Pol Przegl Chir 86: 289-292.[Crossref]

Copyright: (C2016 Elsherif M. This is an open-access article distributed under the terms of the Creative Commons Attribution License, which permits unrestricted use, distribution, and reproduction in any medium, provided the original author and source are credited. 